\title{
A ONE-DIMENSIONAL CONSOLIDATION PROBLEM WITH A MOVING BOUNDARY*
}

\author{
BY \\ R. E. GIBSON \\ (Imperial College, London)
}

1. Introduction. In a previous note [1] some simple one-dimensional diffusion problems of the sphere and infinite cylinder which involve a condition to be satisfied on a specified moving boundary were considered. The progress of consolidation in a clay layer increasing in thickness with time was discussed in a further paper [2]. In these examples it was supposed that the medium vanished at $t=0$, and therefore no initial conditions were prescribed; this restriction was made use of in the method of solution adopted. In many cases of practical consequence, however, a medium of finite extent exists initially through which the required quantity (e.g. temperature, concentration, water pressure) has a given distribution, and in the present note we shall be concerned with a problem of this type, taken from the field of soil mechanics.

2. Consolidation of a clay layer. Consider a layer of homogeneous saturated clay bounded by the planes $x=0$ and $x=h(t)$. The excess water pressure $u$ in the pores of the clay [2] is governed by the equation

$$
c \frac{\partial^{2} u}{\partial x^{2}}=\frac{\partial u}{\partial t}-\gamma \frac{d h}{d t},
$$

where $c$ and $\gamma$ are respectively the coefficient of consolidation and the submerged density of the clay. We seek a solution of (1) subject to the conditions

$$
\begin{aligned}
& \frac{\partial u}{\partial x}(0, t)=0, \quad t>0, \\
& u[h(t), t]=0, \quad t>0,
\end{aligned}
$$

and

$$
u(x, 0)=f(x), \quad 0<x<h(0),
$$

where $h(t)$ and $f(x)$ are given functions.

A direct approach, using for example a Fourier type integral transform, would appear to carry us no further than can the following elementary considerations. A solution of (1) appropriate to the conditions (2) and (4) is clearly

$$
w(x, t)=\gamma[h(t)-h(0)]+(4 \pi c t)^{-1 / 2} \int_{0}^{h(0)} f(\xi) K(x, t ; \xi) d \xi,
$$

where the kernel

$$
K(x, t ; \xi)=\exp \left[-\frac{(x-\xi)^{2}}{4 c t}\right]+\exp \left[-\frac{(x+\xi)^{2}}{4 c t}\right] .
$$

${ }^{*}$ Received February 19, 1959; revised manuscript received June 12, 1959. 
To this we must add a solution to (1) which will enable the requirement (3) to be met while not disturbing conditions (2) and (4). A function possessing the necessary properties is

$$
v(x, t)=-(4 \pi c t)^{-1 / 2} \int_{h(0)}^{\infty} g(\xi) K(x, t ; \xi) d \xi,
$$

since

$$
\begin{aligned}
v(x, 0+) & =-g(x), \quad h(0)<x<\infty \\
& =0, \quad 0<x<h(0),
\end{aligned}
$$

where the function $g$ is the arbitrary element we need to satisfy (3). It is, of course, assumed that the integral (6) exists for $t>0$; this must be verified a posteriori.

The problem therefore reduces from (3), (5) and (6) to the following integral equation

$$
w[h(t), t]=(4 \pi c t)^{-1 / 2} \int_{h(0)}^{\infty} g(\xi) K[h(t), t ; \xi] d \xi
$$

for $g(\xi)$. For reasons which will appear presently it will prove convenient to write

$$
g=g_{1}+g_{2}
$$

and replace (7) by two integral equations, namely

$$
\gamma[h(t)-h(0)]=(4 \pi c t)^{-1 / 2} \int_{h(0)}^{\infty} g_{1}(\xi) K(h, t ; \xi) d \xi
$$

and

$$
\int_{0}^{h(0)} f(\xi) K(h, t ; \xi) d \xi=\int_{h(0)}^{\infty} g_{2}(\xi) K(h, t ; \xi) d \xi .
$$

3. Reduction of the first integral equation. It is unlikely that these equations can be solved for an arbitrary $h(t)$, and we shall be concerned here with the following simple relation

$$
h(t)=a+m t
$$

where $m$, the velocity of this upper boundary, is constant, and $a \geq 0$.

Following Parodi [3] we take the Laplace transform of (8) which becomes

$$
\begin{aligned}
(4 \pi c)^{1 / 2} \gamma m p^{-2}=\int_{a}^{\infty} g_{1}(\xi) \int_{0}^{\infty} t^{-1 / 2} & \exp -\left[p t+\frac{(m t+a-\xi)^{2}}{4 c t}\right] d t d \xi \\
& +\int_{a}^{\infty} g_{1}(\xi) \int_{0}^{\infty} t^{-1 / 2} \exp -\left[p t+\frac{(m t+a+\xi)^{2}}{4 c t}\right] d t d \xi .
\end{aligned}
$$

With the following changes of variable

$$
\begin{aligned}
& \xi=\tau+a, \\
& s=\left(p+\frac{m^{2}}{4 c}\right)^{1 / 2}
\end{aligned}
$$


and

$$
G(\tau)=g_{1}(\tau+a)
$$

and proceeding formally, we find that

$G^{*}\left(\frac{s}{c^{1 / 2}}-\frac{m}{2 c}\right)+G^{*}\left(\frac{s}{c^{1 / 2}}+\frac{m}{2 c}\right) \exp \left[-\left(\frac{2 s}{c^{1 / 2}}+\frac{m}{c}\right) a\right]=2 \gamma m c^{1 / 2} s\left(s^{2}-\frac{m^{2}}{4 c}\right)^{-2}$,

where

$$
G^{*}(\lambda)=\int_{0}^{\infty} e^{-\lambda \tau} G(\tau) d \tau
$$

We now put

$$
z=\frac{s}{c^{1 / 2}}-\frac{m}{2 c}
$$

in (10), which becomes

$G^{*}(z)+G^{*}\left(z+\frac{m}{c}\right) \exp \left[-2 a\left(z+\frac{m}{c}\right)\right]=2 \gamma m c\left(z+\frac{m}{2 c}\right)\left[\left(z+\frac{m}{2 c}\right)^{2} c-\frac{m^{2}}{4 c}\right]^{-2}$,

and in this way the integral equation is reduced to a linear difference equation.

4. Solution of the difference equation. The difference equation may be treated by considering first the solution of (11) with the right hand side zero:

$$
\phi(z)+\phi\left(z+\frac{m}{c}\right) \exp \left[-2 a\left(z+\frac{m}{c}\right)\right]=0 ;
$$

using elementary methods [4] we find

$$
\phi(z)=\exp \left[a z\left(1+\frac{c z}{m}\right)+\frac{i \pi c z}{m}\right]
$$

as a special solution. To obtain the required solution put

$$
G^{*}(z)=\phi(z) \psi(z)
$$

and it follows from (11) that

$$
\psi\left(z+\frac{m}{c}\right)-\psi(z)=-\gamma \frac{m}{c}\left(2 z+\frac{m}{c}\right) z^{-2}\left(z+\frac{m}{c}\right)^{-2} \exp \left\{-\left[a z\left(1+\frac{c z}{m}\right)+\frac{i \pi c}{m} z\right]\right\}
$$

so that, formally at least, a solution is

$$
\begin{aligned}
\psi(z)=\gamma \sum_{n=0}^{\infty}\left\{\left(z+\frac{n m}{c}\right)^{-2}-\right. & {\left.\left[z+(n+1) \frac{m}{c}\right]^{-2}\right\} } \\
\cdot \exp & \left\{-\left[a\left(z+\frac{n m}{c}\right)\left(1+n+\frac{c z}{m}\right)+i \pi\left(n+\frac{c z}{m}\right)\right]\right\} .
\end{aligned}
$$

Using equations (12), (13) and (14) the following expression is obtained

$$
\begin{aligned}
& G^{*}(z)=\gamma \sum_{n=0}^{\infty}(-1)^{n}\left\{\left[z+\frac{n m}{c}\right]^{-2}-\left[z+(n+1) \frac{m}{c}\right]^{-2}\right\} \\
& \cdot \exp \left\{-a n\left[2 z+(n+1) \frac{m}{c}\right]\right\} .
\end{aligned}
$$


5. Solution of the integral equation. The required function $G(\tau)$ of Section 3 can now be found by inverting (15), and we use the result

$$
\frac{1}{2 \pi i} \int_{k-i \infty}^{k+i \infty} e^{2 \tau} e^{-\beta z}(z+\delta)^{-2} d z=\left\{\begin{array}{l}
0, \quad 0 \leq \tau \leq \beta \\
(\tau-\beta) e^{-\delta(\tau-\beta)}, \quad \tau \geq \beta .
\end{array}\right.
$$

By introducing the unit function

$$
\begin{aligned}
U(\tau) & =1, & & \tau>0 \\
& =0, & & \tau<0
\end{aligned}
$$

the solution can be expressed in the succinct form

$$
\begin{aligned}
G(\tau)=\gamma \sum_{n=0}^{\infty}(-1)^{n}(\tau-2 a n)\left\{\exp \left[-\frac{n m}{c}(\tau-2 a n)\right]\right. \\
\left.-\exp \left[-(n+1) \frac{m}{c}(\tau-2 a n)\right]\right\} U(\tau-2 a n) \exp \left[-\frac{a m}{c} n(n+1)\right],
\end{aligned}
$$

so that, finally,

$$
\begin{aligned}
& g_{1}(\xi)=\gamma \sum_{n=0}^{\infty}(-1)^{n}[\xi-(2 n+1) a]\left\{\exp \left[-\frac{n m}{c}(\xi-2 n a-a)\right]\right. \\
& \left.-\exp \left[-(n+1) \frac{m}{c}(\xi-2 n a-a)\right]\right\} U[\xi-(2 n+1) a] \exp \left[-\frac{a m}{c} n(n+1)\right] .
\end{aligned}
$$

The water pressure $u^{\prime}(x, t)$ corresponding to an initial pressure $f(x)=0$ in the layer $0 \leq x \leq a$, the pressures being generated solely by the increasing weight of superincumbent material, is given from (5) and (6) by

$$
u^{\prime}(x, t)=\gamma m t-(4 \pi c t)^{-1 / 2} \int_{a}^{\infty} g_{1}(\xi) K(x, t ; \xi) d \xi .
$$

Rewriting (16) in the form

$$
g_{1}(\xi)=\sum_{n=0}^{\infty} T(\xi, n) U[\xi-(2 n+1) a],
$$

we have

$$
\begin{array}{r}
u^{\prime}=\gamma m t-(4 \pi c t)^{-1 / 2}\left[\int_{a}^{\infty} T(\xi, 0) K(x, t ; \xi) d \xi+\int_{3 a}^{\infty} T(\xi, 1) K(x, t ; \xi) d \xi+\cdots\right] \\
=\gamma m t-(4 \pi c t)^{-1 / 2} \sum_{n=0}^{\infty} \int_{(2 n+1) a}^{\infty} T(\xi, n) K(x, t ; \xi) d \xi
\end{array}
$$

and the integration may be accomplished by introducing the variable

$$
\zeta=\xi-(2 n+1) a
$$

in (17); we find finally that

$$
\begin{aligned}
u^{\prime}=\gamma m t-\gamma(c t)^{1 / 2} \exp \left(-\frac{x^{2}}{4 c t}\right) \sum_{n=0}^{\infty}(-1)^{n}[F(x, t, n)+F(-x, t, n)] \\
\cdot \exp \left\{-\left[\frac{a m}{c} n(n+1)+\frac{a^{2}}{4 c t}(2 n+1)^{2}\right]\right\}
\end{aligned}
$$


where

$$
\begin{aligned}
F(x, t, n) & =\left[\chi\left(\alpha_{2}\right)-\chi\left(\alpha_{1}\right)\right], \\
\chi(\alpha) & =\alpha e^{\alpha^{2}} \operatorname{erfc} \alpha, \\
\alpha_{1} & =(4 c t)^{-1 / 2}[2 n m t+(2 n+1) a-x],
\end{aligned}
$$

and

$$
\alpha_{2}=(4 c t)^{-1 / 2}[2(n+1) m t+(2 n+1) a-x] .
$$

We note here an earlier result [2] for the case $a=0$ :

$$
u^{\prime}(x, t)=\gamma m t-\gamma(\pi c t)^{-1 / 2} \exp \left(-\frac{x^{2}}{4 c t}\right) \int_{0}^{\infty} \xi \tanh \frac{m \xi}{2 c} \cosh \frac{x \xi}{2 c t} \exp \left(\frac{-\xi^{2}}{4 c t}\right) d \xi .
$$

The more general solution (18) with $a=0$ can easily be shown to be equivalent to this if the term sech $(m \xi / 2 c)$ in the above integrand is expanded in exponentials and the series integrated term by term.

6. Reduction of the second integral equation. In the above treatment of the first integral equation (8) we found it possible to reduce the problem to one of determining the solution of a difference equation in the complex plane. The solution of the second integral equation (9) can be found in the same way, but it is instructive to employ a direct method by which the problem is reduced to the solution of a difference equation in the real plane.

We rewrite equation (9) in the equivalent form

$$
\int_{-a}^{a} f(\xi) \exp \left[-(m t+a-\xi)^{2} / 4 c t\right] d \xi=\int_{a}^{\infty} g_{2}(\xi) K(a+m t, t ; \xi) d \xi
$$

where $f(\xi)$ is a specified even function.

We now impose the condition

$$
g_{2}(\xi)=0, \quad-a<\xi<a
$$

and the right hand side of (19) then becomes:

$$
\exp \left(-\frac{m^{2} t}{4 c}\right) \int_{0}^{\infty}\left[g_{2}(\lambda+a) \exp \left(\frac{m \lambda}{2 c}\right)+g_{2}(\lambda-a) \exp \left(-\frac{m \lambda}{2 c}\right)\right] \exp \left(-\frac{\lambda^{2}}{4 c t}\right) d \lambda,
$$

where we have put $\xi=\lambda+a$ in the first term, and $\xi=\lambda-a$ in the second term of the integrand. The left hand side of (19) is now modified by taking

$$
f(\xi)=0, \quad|\xi|>a,
$$

and then

$$
\begin{aligned}
\int_{-a}^{a} f(\xi) \exp \left[-(m t+a-\xi)^{2} / 4 c t\right] d \xi=\int_{-\infty}^{a} f(\xi) & \exp \left[-(m t+a-\xi)^{2} / 4 c t\right] d \xi \\
= & \int_{0}^{\infty} f(\lambda-a) \exp \left[-(m t+\lambda)^{2} / 4 c t\right] d \lambda
\end{aligned}
$$


where $\lambda=a-\xi$. The equation (19) now takes the form

$\int_{0}^{\infty}\left[g_{2}(\lambda+a) \exp \left(\frac{m \lambda}{2 c}\right)+\left\{g_{2}(\lambda-a)-f(\lambda-a)\right\} \exp \left(-\frac{m \lambda}{2 c}\right)\right] \exp \left(-\frac{\lambda^{2}}{4 c t}\right) d \lambda=0$

for all values of $t>0$. It is natural to assume that the integrand vanishes and this leads to the following difference equation

$$
g_{2}(\lambda+a) \exp (m \lambda / c)+g_{2}(\lambda-a)=f(\lambda-a) .
$$

The condition (20) enables $g_{2}$ to be determined uniquely. We omit the details of the solution which is

$$
g_{2}(\xi)=(-1)^{n+1} f(\xi-2 n a) \exp [-n m(\xi-n a) / c] ; \quad(2 n-1) \leq \xi \leq(2 n+1) .
$$

From (5), (6) and (22) the water pressure $u^{\prime \prime}(x, t)$ in the layer $0 \leq x \leq a+m t$, when the initial pressure is $f(x)$ in $0 \leq x \leq a$ and when the accreting material is supposed weightless $(\gamma=0)$, is

$$
\begin{gathered}
u^{\prime \prime}(x, t)=(4 \pi c t)^{-1 / 2} \int_{-o}^{a} f(\xi) \exp \left[-(x-\xi)^{2} / 4 c t\right] d \xi \\
-(4 \pi c t)^{-1 / 2} \sum_{n=1}^{\infty}(-1)^{n+1} \int_{-a}^{a} f(\xi) K(x, t ; \xi+2 n a) \exp [-n m(\xi+n a) / c] d \xi .
\end{gathered}
$$

The complete solution to the problem of Section 2 is now found from the sum of (18) and (23)

$$
u=u^{\prime}+u^{\prime \prime}
$$

It can easily be verified that equations (1)-(4) are satisfied and that the series involved in the solution converge for $t>0$ and $f$ bounded.

7. Some alternative boundary conditions. When other boundary conditions are imposed the use of the above method depends upon our being able to choose a solution at the outset which satisfies all conditions except that on the moving boundary; in simple cases a knowledge of the appropriate Green's functions will be sufficient. For example, to satisfy (1) with initial condition (4) and boundary conditions

$$
\begin{gathered}
u[h(t), t]=\psi(t) \\
u(0, t)=\phi(t)
\end{gathered}
$$

we should take as a tentative solution

$$
\begin{aligned}
u=\gamma[h(t)-h(0)]-x(4 \pi c)^{-1 / 2} & \int_{0}^{t} \frac{[\gamma h(\tau)-\gamma h(0)-\phi(\tau)]}{(t-\tau)^{3 / 2}} \exp \left[-\frac{x^{2}}{4 c(t-\tau)}\right] d \tau \\
& +\int_{h(0)}^{\infty} g(\xi) Q(x, t ; \xi) d \xi+\int_{0}^{h(0)} f(\xi) Q(x, t ; \xi) d \xi,
\end{aligned}
$$

where

$$
Q(x, t ; \xi)=(4 \pi c t)^{-1 / 2}\left\{\exp \left[-(x-\xi)^{2} / 4 c t\right]-\exp \left[-(x+\xi)^{2} / 4 c t\right]\right\}
$$


our choice being dictated by the nature of the initial and boundary conditions. The expression (26) satisfies (1), (4) and (25) and the function $g$ is to be found from the condition (24); this requires us to solve the linear equation

$$
\begin{aligned}
\psi(t)-\gamma[h(t)-h(0)]+h(t)(4 \pi c)^{-1 / 2} & \int_{0}^{t} \frac{[\gamma h(\tau)-\gamma h(0)-\phi(\tau)]}{(t-\tau)^{3 / 2}} \exp \left[-\frac{h^{2}(t)}{4 c(t-\tau)}\right] d \tau \\
& -\int_{0}^{h(0)} f(\xi) Q[h(t), t ; \xi] d \xi=\int_{h(0)}^{\infty} g(\xi) Q[h(t), t ; \xi] d \xi,
\end{aligned}
$$

and with $h(t)$ of the form (10) the solution may be found along the lines adopted above.

\section{Biblography}

1. R. E. Gibson, $A$ heat conduction problem involving a specified moving boundary, Quart. Appl. Math. 16, 426-430 (1959)

2. R. E. Gibson, The progress of consolidation in a clay layer increasing in thickness with time, Géotechnique 8, 171-182 (1958)

3. M. Parodi, Équations Intégrales et Transformation de Laplace, Publ. Sci. et Tech. du Ministère de L'Air. No. 242, Paris, 1950, p. 67

4. See, for example, L. M. Milne-Thomson, The Calculus of Finite Differences, Macmillan, 1933, p. 328 